\title{
Properties of continuous Petri nets controlled via model predictive control
}

\author{
Alessandro Giua, Cristian Mahulea, Laura Recalde, Carla Seatzu, Manuel Silva
}

\begin{abstract}
In previous papers we investigated the problem of deriving an optimal control law for continuous Petri nets under infinite server semantics. In particular, the problem was studied through Model Predictive Control (MPC), an advanced control method, extensively used in industrial applications.

In this work we discuss some properties of the resulting closed-loop system, such as feasibility and asymptotic stability. We prove that for continuous Petri nets feasibility is always guaranteed, while asymptotic stability is not ensured. Finally, we demonstrate that the introduction of an appropriate terminal constraint may guarantee asymptotic stability provided that certain assumptions on the initial state and the moving horizon are satisfied.
\end{abstract}

\section{INTRODUCTION}

Discrete Petri nets (PN) are a mathematical formalism for the description of discrete-event systems, successfully used for modeling, analysis and synthesis of such systems. Its main feature is that their state space belongs to the set of non-negative integers [5]. Another key feature of PN is their capacity to represent graphically and visualize primitives such as parallelism, synchronization, mutual exclusion, etc.

As other models of concurrent systems, discrete PN may suffer from the state explosion problem. As a consequence the analysis and optimization of these systems may require an amount of computational effort, thus leading to analytically and computationally untractable problems. One way to tackle this difficulty consists in the relaxation of the original integrality constraints, giving a fluid (i.e., continuous) approximation of the discrete event dynamics [5], [14]. Fluid models may be studied by means of structural analysis, that does not require the enumeration of the state space [14].

To study the performance of systems, timing can be introduced and timed PN are obtained. In this paper we consider timed continuous Petri net systems (contPN) under infinite server semantics and subject to external control actions: we assume that the only admissible control law consists in

This work was partially supported by the projects CICYT - FEDER DPI2003-06376 and DPI2006-15390.

A. Giua and C. Seatzu are with the Department of Electrical and Electronic Engineering, University of Cagliari, Piazza D’Armi, 09123 Cagliari, Italy $\{$ giua, seatzu\}@diee.unica.it.

C. Mahulea, L. Recalde and M. Silva are with the Department of Computer Science and Systems Engineering, University of Zaragoza, Maria de Luna 1, 50018 Zaragoza Spain, $\{$ cmahulea, lrecalde, silva\}eunizar.es. slowing down the firing speed of transitions [14]. Such a system can be represented by a particular hybrid positive model: a piecewise linear positive model with autonomous switches and with constraints on the state and control input space [10]. By a suitable change of variables it is also possible, as shown in [9], to further simplify the model into a discrete-time linear model with constraints on the state and input space.

In the case of discrete event systems (DES), the problem of on-line calculation of supervisory controllers has been introduced in [4] where the framework of limited lookahead control policies is used. For DES modeled by PN, in [6] an integer linear programming formalism is used while, for a particular class of marked graphs, in [12] a control strategy based on Model Predictive Control (MPC) has been studied.

For contPN systems with infinite server semantics, in [7] we studied the problem of deriving an optimal control law that enables the system to reach a desired point starting from a given initial marking, while optimizing a quadratic performance index. In particular, we assumed that the desired marking and control was a steady state point [10], namely a stable operation point where the system can work indefinitely.

The solution proposed in [7] is based on MPC [3]. In particular, in [7] we investigated the possibility of using both an implicit and an explicit [1] MPC control strategy. A detailed comparison among the two approaches is also proposed in [7] in terms of computational complexity and practical implementation.

The contribution of this paper consists in the discussion of some properties of the system controlled via MPC, such as feasibility and asymptotic stability. We prove that for contPN systems feasibility is always guaranteed, while asymptotic stability is not ensured. Different approaches are investigated in order to guarantee this property. One of them consists in the introduction of an appropriate terminal constraint, and in such a case asymptotic stability can be guaranteed under certain assumptions on the initial state and on the moving horizon.

Some numerical examples are also presented to clearly show that, although some of the assumptions may look conservative, they cannot be relaxed when the desired (final) state and input is not an interior point to the set of feasible 
states and inputs, but lies on the boundary.

\section{Continuous Petri nets}

Definition 2.1: A contPN system is a pair $\left\langle\mathcal{N}, \boldsymbol{m}_{\mathbf{0}}\right\rangle$, where: $\mathcal{N}=\langle P, T$, Pre, Post $\rangle$ is the net structure with set of places $P$, set of transitions $T$, pre and post incidence matrices Pre, Post $: P \times T \rightarrow \mathbb{N} ; \boldsymbol{m}_{\mathbf{0}}: P \rightarrow \mathbb{R}_{\geq 0}$ is the initial marking.

We denote $\boldsymbol{m}(\tau)$ the marking at time $\tau$ and in discrete time we denote $\boldsymbol{m}(k)$ the marking at sampling instant $k$, where $\tau=k \cdot \Theta$ and $\Theta$ is the sampling period. Finally, the preset and postset of a node $x \in P \cup T$ are denoted ${ }^{\bullet} x$ and $x^{\bullet}$, respectively.

A transition $t \in T$ is enabled at $\boldsymbol{m}$ iff $\forall p_{i} \in{ }^{\bullet} t, m_{i}>0$, and its enabling degree is

$$
\operatorname{enab}(t, \boldsymbol{m})=\min _{p_{i} \in \bullet}\left\{\frac{m_{i}}{\operatorname{Pre}\left(p_{i}, t\right)}\right\} .
$$

An enabled transition $t$ can fire in any real amount $0 \leq \alpha \leq$ $\operatorname{enab}(t, \boldsymbol{m})$ leading to a new marking $\boldsymbol{m}^{\prime}=\boldsymbol{m}+\alpha \boldsymbol{C}(\cdot, t)$, where $\boldsymbol{C}=$ Post-Pre is the token flow matrix; this firing is also denoted $\boldsymbol{m}[t(\alpha)\rangle \boldsymbol{m}^{\prime}$.

In general, if $\boldsymbol{m}$ is reachable from $\boldsymbol{m}_{\mathbf{0}}$ through a sequence $\sigma=t_{r_{1}}\left(\alpha_{1}\right) t_{r_{2}}\left(\alpha_{2}\right) \ldots t_{r_{k}}\left(\alpha_{k}\right)$, and we denote by $\boldsymbol{\sigma}$ : $T \rightarrow \mathbb{R}_{\geq 0}$ the firing vector whose component associated to a transition $t_{j}$ is

$$
\sigma_{j}=\sum_{h \in H\left(\sigma, t_{j}\right)} \alpha_{h}
$$

where

$$
H\left(\sigma, t_{j}\right)=\left\{h=1, \ldots, k \mid t_{r_{h}}=t_{j}\right\},
$$

we can write:

$$
\boldsymbol{m}=\boldsymbol{m}_{0}+C \cdot \sigma
$$

which is called the fundamental equation.

The basic difference between discrete and continuous PN is that the components of the markings and firing count vectors are not restricted to take values in the set of natural numbers but can take non-negative real values.

Definition 2.2: A (deterministically) timed contPN system is a contPN system together with a vector $\lambda: T \rightarrow \mathbb{R}_{>0}$, where $\lambda_{j}$ is the firing rate of $t_{j}$.

Now, the fundamental equation depends on time: $\boldsymbol{m}(\tau)=$ $\boldsymbol{m}_{\mathbf{0}}+\boldsymbol{C} \cdot \boldsymbol{\sigma}(\tau)$, where $\boldsymbol{\sigma}(\tau)$ denotes the firing count vector in the interval $[0, \tau]$. Differentiating it with respect to time the following is obtained: $\dot{\boldsymbol{m}}(\tau)=\boldsymbol{C} \cdot \dot{\boldsymbol{\sigma}}(\tau)$. The derivative of firing vector represents the flow of the timed model $\boldsymbol{f}(\tau)=\dot{\boldsymbol{\sigma}}(\tau)$. Depending on how the flow of the transition is defined, many firing semantics are possible [5], [14]. This paper deals with infinite server semantics that for a broad class of systems approximates better the behavior of the underlying discrete net [11]. Under this semantics, the flow of transition $t_{j}$ is given by:

$$
f_{j}=\lambda_{j} \operatorname{enab}\left(t_{j}, m\right)=\lambda_{j} \min _{p_{i} \in t_{j}}\left\{\frac{m_{i}}{\operatorname{Pre}\left(p_{i}, t_{j}\right)}\right\} .
$$

\section{A LINEAR DISCRETE-TIME CONSTRAINED MODEL}

In this section we consider net systems subject to external control actions, and assume that the only admissible control law consists in slowing down the firing speed of transitions, that are assumed to be all controllable [10].

Definition 3.1: The flow of the forced (or controlled) timed contPN will be denoted $\boldsymbol{w}(\tau)=\boldsymbol{f}(\tau)-\boldsymbol{u}(\tau)$, where the external control $\boldsymbol{u}(\tau)$ satisfies $\mathbf{0} \leq \boldsymbol{u}(\tau) \leq \boldsymbol{f}(\tau)$.

Therefore, the control input will be dynamically upper bounded by the flow of the corresponding unforced system.

The overall behavior of the system is ruled by the following system

$$
\left\{\begin{array}{l}
\dot{\boldsymbol{m}}(\tau)=\boldsymbol{C} \cdot[\boldsymbol{f}(\tau)-\boldsymbol{u}(\tau)] \\
0 \leq \boldsymbol{u}(\tau) \leq \boldsymbol{f}(\tau)
\end{array}\right.
$$

This is a particular hybrid system: a piecewise linear system with autonomous switches and dynamic (or statebased) constraints in the input.

Proposition 3.2: [9] Any piecewise linear constrained model of the form (1) can be rewritten as a linear constrained model of the form

$$
\left\{\begin{array}{l}
\dot{\boldsymbol{m}}(\tau)=\boldsymbol{C} \cdot \boldsymbol{w}(\tau) \\
\boldsymbol{G} \cdot\left[\begin{array}{l}
\boldsymbol{w}(\tau) \\
\boldsymbol{m}(\tau)
\end{array}\right] \leq \mathbf{0} \\
\boldsymbol{w}(\tau) \geq \mathbf{0}
\end{array}\right.
$$

that we call continuous time contPN model (or ct-contPN for short) where $\boldsymbol{G}$ is an appropriate constant matrix defined as follows:

$$
\begin{gathered}
\boldsymbol{G}=\left[\begin{array}{ll}
\boldsymbol{\Delta} & -\boldsymbol{\Gamma}
\end{array}\right], \quad \boldsymbol{\Delta} \in \mathbb{Z}^{q \times|T|}, \quad \boldsymbol{\Gamma} \in \mathbb{Z}^{q \times|P|}, \\
q=\sum_{t \in T}|\bullet t|,
\end{gathered}
$$

and the row of $\boldsymbol{\Delta}$ and $\boldsymbol{\Gamma}$ relative to the generic pre $\operatorname{arc}\left(p_{i}, t_{j}\right)$ are

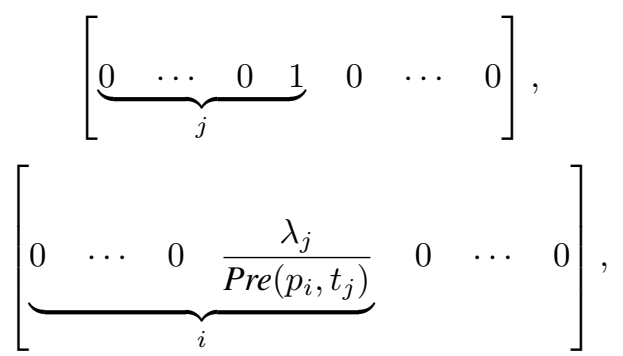

respectively. The initial value of the state of this system is $\boldsymbol{m}(0)=\boldsymbol{m}_{\mathbf{0}} \geq \mathbf{0}$.

The system in eq. (2) is a linear system with a state-matrix equal to $\mathbf{0}$ and an input matrix equal to the token flow matrix of the contPN. There is still a dynamic constraint on the 


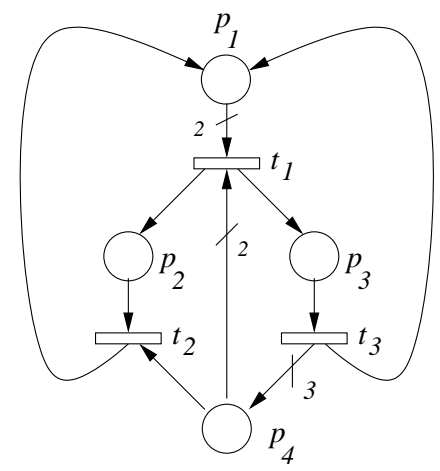

Fig. 1. A simple contPN.

system inputs that depends on the value of the system state $\boldsymbol{m}$. The continuous-time system (2) can be discretized, thus obtaining a discrete-time "equivalent" model.

Definition 3.3: Consider a ct-contPN model of the form (2) and let $\Theta$ be a sampling period. A model can be given in terms of a discrete-time contPN or $d t$-contPN as follows:

$$
\left\{\begin{array}{l}
\boldsymbol{m}(k+1)=\boldsymbol{m}(k)+\Theta \cdot \boldsymbol{C} \cdot \boldsymbol{w}(k) \\
\boldsymbol{G} \cdot\left[\begin{array}{c}
\boldsymbol{w}(k) \\
\boldsymbol{m}(k)
\end{array}\right] \leq \mathbf{0} \\
\boldsymbol{w}(k) \geq \mathbf{0}
\end{array}\right.
$$

The initial value of the state of this system is $\boldsymbol{m}(0)=\boldsymbol{m}_{\mathbf{0}} \geq$ 0.

It is important to stress that, although the evolution of a dt-contPN occurs in discrete steps, as was the case for an untimed system, discrete time evolutions and untimed evolutions are not the same. In fact, while an untimed net system can be seen evolving sequentially, executing a single transition firing at each step, a dt-contPN may evolve in concurrent steps where more than one transition can fire.

In a ct-contPN under infinite servers semantics, the positiveness of the marking is ensured if the initial marking $\boldsymbol{m}_{\mathbf{0}}$ is positive, because the flow of a transition goes to zero whenever one of the input places is empty [14].

In a dt-contPN, this is not always true as it is illustrated in [9]. In the same work it is proved that if the sampling period is small enough the problem can be avoided. In the rest of the paper we will always implicitly assume that $\Theta$ is small enough and all reachable markings are positive.

\section{OPTIMAL TRANSIENT CONTROL VIA MPC}

Steady state optimal control of contPN was studied in [10] and if all transitions can be controlled and the objective function is linear, the problem can be solved in polynomial time. The solution is an optimal marking and an optimal control input in steady state.

In [7] we assumed that the steady state condition $\left(\boldsymbol{m}_{\boldsymbol{f}}\right.$, $\boldsymbol{w}_{\boldsymbol{f}}$ ) was known and our problem was how to reach it (from a given $\boldsymbol{m}_{\mathbf{0}}$ ) in a finite time while optimizing a given performance index. This steady state condition can be an optimal one or any other reachable marking together with a command. The optimal control was studied using Model Predictive Control (MPC) [8], also referred as moving horizon control or receding horizon control.

MPC algorithms use different cost functions to obtain the control action. In [7] we considered the following standard quadratic form:

$$
\begin{aligned}
& J(\boldsymbol{m}(k), \boldsymbol{w}(k), N)= \\
& \quad\left\{\left(\boldsymbol{m}(k+N)-\boldsymbol{m}_{f}\right)^{\prime} \cdot \boldsymbol{Z} \cdot\left(\boldsymbol{m}(k+N)-\boldsymbol{m}_{f}\right)\right. \\
& \quad+\sum_{j=0}^{N-1}\left[\left(\boldsymbol{m}(k+j)-\boldsymbol{m}_{f}\right)^{\prime} \cdot \boldsymbol{Q} \cdot\left(\boldsymbol{m}(k+j)-\boldsymbol{m}_{f}\right)+\right. \\
& \left.\left.\quad\left(\boldsymbol{w}(k+j)-\boldsymbol{w}_{f}\right)^{\prime} \cdot \boldsymbol{R} \cdot\left(\boldsymbol{w}(k+j)-\boldsymbol{w}_{f}\right)\right]\right\}
\end{aligned}
$$

where $Z, Q$ and $R$ are positive definite matrices.

The constraints are derived from the dt-contPN definition, and at every step the new marking should respect (3). Thus, at each step the following problem needs to be solved:

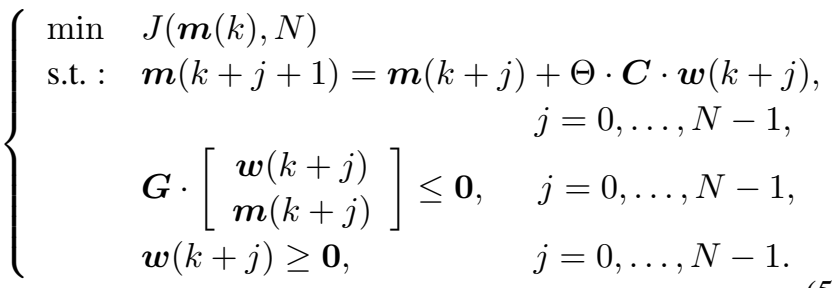

We denote as implicit MPC the MPC control law computed solving on-line the optimization problem (5).

An alternative to implicit MPC has been proposed in [1]. Here the authors present a technique to compute off-line an explicit solution of the MPC control problem, based on multi-parametric linear programming (mp-LP) or quadratic programming (mp-QP). They split the maximum controllable set (i.e., all states that are controllable) into polytopes described by linear inequalities ${ }^{1}$ in which the control command is described as a piecewise affine function of the state. Thus, the control law results in a state feedback control law.

In [7] we applied both the two MPC approaches to dtcontPN. Detailed comparisons among them are reported in [7]. In particular, several numerical simulations have been carried out using different values of $N$ and/or $\Theta$. We also observed that if the considered final input lays on the boundary of the region of feasible state + input vectors, the infinite horizon problem $(N=+\infty)$ cannot be solved. In fact, in this case it is not possible to determine a finite value of $N$ in order to reduce the problem to a finite time optimal control problem.

\footnotetext{
${ }^{1}$ A bounded polyhedron $\mathcal{P} \subset \mathbb{R}^{n}, \mathcal{P}=\left\{\boldsymbol{x} \in \mathbb{R}^{n} \quad \mid \boldsymbol{A x} \leq \boldsymbol{B}\right\}$ is called a polytope.
} 


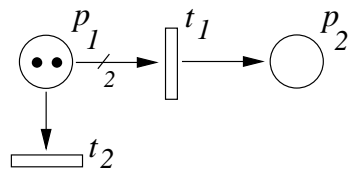

Fig. 2. Example of an unstable contPN system with basic MPC scheme.

\section{PRoperties of THE ClOSED-LOOP SYSTEM}

In this section we investigate some properties of the closed-loop system (resulting from the application of MPC), such as feasibility and asymptotic stability.

\section{A. Feasibility}

In general, given an initial feasible state, there is no guarantee that the optimization problem we need to solve at each time step when implementing implicit MPC, will remain feasible at all future time steps $k$, as the system might enter "blind alleys" where no solution to the optimization problem exists [2]. In terms of explicit MPC this translates into the fact that there is no guarantee that the resulting state space partition includes all feasible states.

However, thanks to the structure of the constraints, in the case of continuous Petri net systems the following result guarantees the feasibility of (5) for any time step $k$.

Proposition 5.1: The optimization problem (5) is feasible for any $\boldsymbol{m}(k) \geq \mathbf{0}$.

Proof: The solution $\boldsymbol{w}(k+j)=\mathbf{0}$ for $j=0,1, \ldots, N-$ 1 is feasible. In fact,

$$
\begin{aligned}
\boldsymbol{G} \cdot\left[\begin{array}{l}
\boldsymbol{w}(k+j) \\
\boldsymbol{m}(k+j)
\end{array}\right] \\
\quad=\left[\begin{array}{ll}
\boldsymbol{\Delta}-\boldsymbol{\Gamma}
\end{array}\right] \cdot\left[\begin{array}{ll}
\boldsymbol{w}(k+j) & \boldsymbol{m}(k+j)
\end{array}\right] \\
\quad=-\boldsymbol{\Gamma} \cdot \boldsymbol{m}(k+j) \leq \mathbf{0}
\end{aligned}
$$

because (see Proposition 3.2) $\boldsymbol{\Gamma}$ is a matrix of non-negative numbers and $\boldsymbol{m}(k+j)=\boldsymbol{m}_{k} \geq \mathbf{0}$ for any $j=0,1, \ldots, N-$ 1 .

In simple words Proposition 5.1 claims that feasibility is guaranteed for any $\boldsymbol{m}(k) \geq \mathbf{0}$ because we can always "stop" the net.

\section{B. Asymptotic stability}

The feasibility of (5) is obviously a desirable property but it does not ensure the convergence of the optimal solution to the desired state, that is our main requirement.

The following example clearly shows this.

Example 5.2: Let us consider the net system in Fig. 2 with $\boldsymbol{\lambda}=\left[\begin{array}{ll}1 & 5\end{array}\right]^{T}$. Let $\Theta=0.1, \boldsymbol{m}_{f}=\left[\begin{array}{ll}0 & 1\end{array}\right]^{T}$ and $\boldsymbol{w}_{f}=$ $\left[\begin{array}{ll}0 & 0\end{array}\right]^{T}$. Moreover, let $\boldsymbol{Q}=\boldsymbol{Z}=\boldsymbol{R}=\boldsymbol{I}$ and $N=1$.

The marking evolution of the system controlled with the MPC policy is presented in Fig. 3. Clearly, the desired marking is not reached. Observe that to obtain $\boldsymbol{m}_{f}$, only $t_{1}$ should fire. Because the timing horizon is too short and
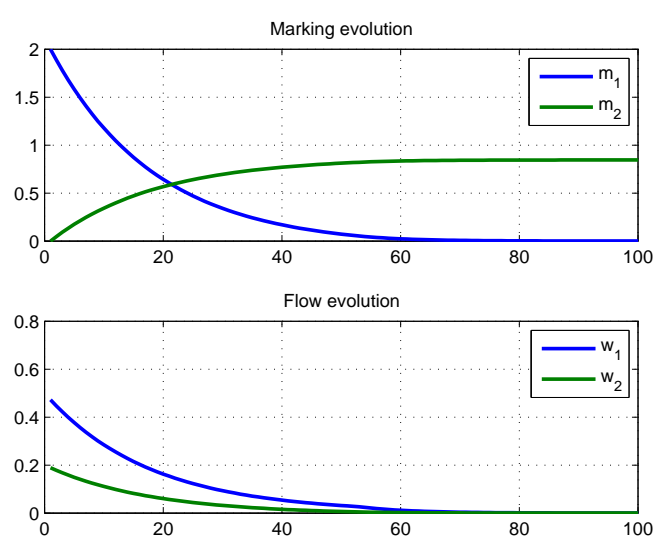

Fig. 3. Marking and flow evolution of the contPN system in Fig. 2.

$\lambda_{2}=5>>\lambda_{1}=1$, the optimality of (5) implies that it is better to fire at the beginning "a little" $t_{2}$ so that $m_{1}$ approaches the desired final value $m_{f, 1}=0$. However, once $t_{2}$ has fired, $\boldsymbol{m}_{f}$ cannot be reached because there is not enough marking in $p_{1}$ to be transferred to $p_{2}$.

In this section we investigate three different approaches in order to improve convergence, that are quite standard in the literature [2].

B1. The first approach consists in assuming that

$$
\boldsymbol{w}(k+j)=\mathbf{0} \quad \forall j=N+1, \cdots, \infty
$$

and weighting the distance of the marking from the final one not only for $j=0,1, \cdots, N-1$ but for any $j=0,1, \cdots, \infty$.

Obviously, such an approach can only be applied to asymptotically stable systems, that is not the case here because the dynamical matrix is the identity one.

B2. The second approach consists in assuming that

$$
\boldsymbol{w}(k+j)=\boldsymbol{K} \boldsymbol{m}(k+j) \quad \forall j=N+1, \cdots, \infty
$$

and weighting the distance of the marking from the final marking not only for $j=0,1, \cdots, N-1$ but for any $j=0,1, \cdots, \infty$. In particular, we assume that matrix $\boldsymbol{K}$ is defined as in the unconstrained LQR problem with weighting matrices $\boldsymbol{Q}$ and $\boldsymbol{R}$, namely

$$
\begin{aligned}
& \boldsymbol{K}=-\left(\boldsymbol{R}+\boldsymbol{B}^{T} \boldsymbol{K} \boldsymbol{B}\right)^{-1} \boldsymbol{B}^{T} \boldsymbol{P} \boldsymbol{A}, \\
& \left.\boldsymbol{P}=(\boldsymbol{A}+\boldsymbol{B} \boldsymbol{K})^{T} \boldsymbol{P}(\boldsymbol{A}+\boldsymbol{B} \boldsymbol{K})\right)+\boldsymbol{K}^{T} \boldsymbol{R} \boldsymbol{K}+\boldsymbol{Q},
\end{aligned}
$$

and in our case $\boldsymbol{A}=\boldsymbol{I}$ and $\boldsymbol{B}=\boldsymbol{\Theta} \cdot \boldsymbol{C}$.

In plain words we apply an open-loop optimal strategy just for the first $N$ steps; this leads us close enough to the optimal desired state and then we can switch to a LQR optimal regulator without violating the constraints.

This is equivalent to consider an optimization problem of the form (5) where the matrix $Z$ in the performance index is $\boldsymbol{Z}=\boldsymbol{P}$, where $\boldsymbol{P}$ is defined as in eq. (6). 
In such a case, using results from the classical optimal control theory [13], we can guarantee convergence to the desired condition only if the set of feasible state and input vectors is bounded and the final state and input is an interior point of it. As a consequence such an approach often does not apply to many control problems within the framework of continuous Petri nets, where is frequently the case of steady state input on the border. Thus we need to investigate for alternative approaches.

Note however that, if the final state + input is an interior point, and the moving horizon $N$ is sufficiently large, this approach is surely the most convenient. In fact, it has the major advantage that the resulting strategy is indeed the optimal infinite horizon constrained LQR policy [2].

B3. The third approach we consider consists in forcing the system state at time $k+N$ to belong to the straight line $\boldsymbol{m}(k)-\boldsymbol{m}_{f}$. In simple words, this is equivalent to add a terminal constraint of the form

$$
\left\{\begin{array}{l}
\boldsymbol{m}(k+N)=\boldsymbol{m}_{k}+\alpha \cdot\left(\boldsymbol{m}_{f}-\boldsymbol{m}(k)\right) \\
0 \leq \alpha \leq 1
\end{array}\right.
$$

to the optimization problem (5), where $\alpha$ is a new decision variable.

A remark should be done. We observe that the addition of this constraint makes it necessary to solve a certain number of bilinear (rather than linear) programming problems when implementing the explicit MPC [2]. In particular, bilinear problems have to be solved when computing the Chebychev centers of the polytopic regions, where both the initial state and $\alpha$ are unknown.

This approach revealed satisfactory in several numerical examples we considered. However, we have been able to prove convergence only under certain assumptions, as detailed in the following.

Proposition 5.3: Let us consider a contPN system. Let $\boldsymbol{m}_{\mathbf{0}}$ and $\boldsymbol{m}_{\boldsymbol{f}}$ be the initial and final markings, respectively, with $\boldsymbol{m}_{\mathbf{0}}>\mathbf{0}$ and $\boldsymbol{m}_{\boldsymbol{f}}$ reachable from $\boldsymbol{m}_{\mathbf{0}}$. Assume that the system is controlled using MPC with a terminal constraint of the form (7) and prediction horizon $N=1$. Then the closed-loop system is asymptotically stable.

Proof: We prove the statement in three steps. We first prove that if $\boldsymbol{m}_{\mathbf{0}}>\mathbf{0}$ then $\alpha>0$ in eq. (7) is feasible at any $k \geq 0$. Then, we define a quadratic function that we prove to be a Lyapunov function. Finally, we demonstrate that it is strictly decreasing.

- We first observe that by item (2) of Proposition 4.4 in [9], if $\boldsymbol{m}_{\mathbf{0}}>\mathbf{0}$ then $\boldsymbol{m}(k)>\mathbf{0}$ for any $k \geq 1$. Moreover, if $\boldsymbol{m}_{\boldsymbol{f}}$ is reachable from $\boldsymbol{m}_{\mathbf{0}}$ then it is also reachable from any marking in the straight path $\boldsymbol{m}_{\boldsymbol{f}}-\boldsymbol{m}_{\mathbf{0}}$ being the full reachability space a convex region.

Now, let us consider eq. (7) with $N=1$. It holds $\boldsymbol{m}(k+$ $1)=\alpha \cdot \boldsymbol{m}_{\boldsymbol{f}}+(1-\alpha) \cdot \boldsymbol{m}(k)$. Being $\boldsymbol{m}_{\boldsymbol{f}}$ reachable from $\boldsymbol{m}(k)$, then there exists $\boldsymbol{\sigma} \geq \mathbf{0}$ such that $\boldsymbol{m}_{\boldsymbol{f}}=\boldsymbol{m}(k)+\boldsymbol{C} \cdot \boldsymbol{\sigma}$.

Thus, $\boldsymbol{m}(k+1)=\alpha \cdot \boldsymbol{m}(k)+\alpha \cdot \boldsymbol{C} \cdot \boldsymbol{\sigma}+(1-\alpha) \cdot \boldsymbol{m}(k)=$ $\boldsymbol{m}(k)+\boldsymbol{C} \cdot(\alpha \boldsymbol{\sigma})$. But there always exists $\alpha>0$ such that $\alpha \boldsymbol{\sigma}$ can always be fired at $\boldsymbol{m}(k)$ being $\boldsymbol{m}(k)>\mathbf{0}$.

- Now, without loss of generality we assume that in eq. (5) it holds: (a) $\boldsymbol{m}_{\boldsymbol{f}}=\mathbf{0}$; (b) $\boldsymbol{C}$ is full rank. In fact, if $\boldsymbol{m}_{\boldsymbol{f}}=\mathbf{0}$ we can always redefine the state in eq. (5) by translation. Furthermore, if $\boldsymbol{C}$ is not full rank then there exist vectors in the null-space of $\boldsymbol{C}$ (called P-flows of the net) that impose some invariants on the state space: this allows one to reduce the dimension of the state vector in eq. (5) until a full rank matrix $C$ is obtained.

Let

$$
V(\boldsymbol{m}(k))=\boldsymbol{m}(k)^{T} \cdot \boldsymbol{Z} \cdot \boldsymbol{m}(k)
$$

where $Z$ is the weighting matrix in the performance index (4).

Obviously, $V(\boldsymbol{m}(k)) \geq 0$ for any $\boldsymbol{m}(k) \neq \mathbf{0}$, being $\boldsymbol{Z}$ positive definite. Moreover, $V(\boldsymbol{m}(k+1)) \leq V(\boldsymbol{m}(k))$ for any $k \geq 0$. In fact, under the assumption that $\boldsymbol{m}_{\boldsymbol{f}}=\mathbf{0}$, by constraint (7) it holds $\boldsymbol{m}(k+1)=(1-\alpha) \cdot \boldsymbol{m}(k)$. Thus,

$$
\begin{aligned}
V(\boldsymbol{m}(k+1)) & =\boldsymbol{m}(k+1)^{T} \cdot \boldsymbol{Z} \cdot \boldsymbol{m}(k+1) \\
& =(1-\alpha)^{2} \cdot \boldsymbol{m}(k)^{T} \cdot \boldsymbol{Z} \cdot \boldsymbol{m}(k) \\
& =(1-\alpha)^{2} \cdot V(\boldsymbol{m}(k)) \leq V(\boldsymbol{m}(k)) .
\end{aligned}
$$

- We now prove that $\forall k \geq 0$ the optimal solution of problem (5) leads to $\alpha>0$. Let $k$ be an arbitrary time instant. If $\alpha=0$ then the performance index (4) is equal to

$$
J^{\prime}=\boldsymbol{m}(k)^{T} \cdot \boldsymbol{Q} \cdot \boldsymbol{m}(k)+\boldsymbol{m}(k)^{T} \cdot \boldsymbol{Z} \cdot \boldsymbol{m}(k) .
$$

If $\alpha>0$ (this is always possible by the first item of this proof), then the performance index (4) is

$$
\begin{aligned}
J^{\prime \prime}= & \boldsymbol{m}(k)^{T} \cdot \boldsymbol{Q} \cdot \boldsymbol{m}(k)+\boldsymbol{w}(k)^{T} \cdot \boldsymbol{R} \cdot \boldsymbol{w}(k)+ \\
& +\boldsymbol{m}(k+1)^{T} \cdot \boldsymbol{Z} \cdot \boldsymbol{m}(k+1) .
\end{aligned}
$$

Being $\boldsymbol{m}(k+1)=(1-\alpha) \cdot \boldsymbol{m}(k)$, it holds

$$
\begin{aligned}
J^{\prime \prime}= & \boldsymbol{m}(k)^{T} \cdot \boldsymbol{Q} \cdot \boldsymbol{m}(k)+\boldsymbol{w}(k)^{T} \cdot \boldsymbol{R} \cdot \boldsymbol{w}(k) \\
& +\boldsymbol{m}(k)^{T} \cdot \boldsymbol{Z} \cdot \boldsymbol{m}(k)-2 \cdot \alpha \cdot \boldsymbol{m}(k)^{T} \cdot \boldsymbol{Z} \cdot \boldsymbol{m}(k) \\
& +\alpha^{2} \cdot \boldsymbol{m}(k)^{T} \cdot \boldsymbol{Z} \cdot \boldsymbol{m}(k)
\end{aligned}
$$

and

$$
\begin{aligned}
J^{\prime \prime}-J^{\prime}= & \boldsymbol{w}(k)^{T} \cdot \boldsymbol{R} \cdot \boldsymbol{w}(k)+\alpha^{2} \cdot \boldsymbol{m}(k)^{T} \cdot \boldsymbol{Z} \cdot \boldsymbol{m}(k) \\
& -2 \cdot \alpha \cdot \boldsymbol{m}(k)^{T} \cdot \boldsymbol{Z} \cdot \boldsymbol{m}(k) \\
= & \boldsymbol{w}(k)^{T} \cdot \boldsymbol{R} \cdot \boldsymbol{w}(k) \\
& +\alpha \cdot(\alpha-2) \cdot \boldsymbol{m}(k)^{T} \cdot \boldsymbol{Z} \cdot \boldsymbol{m}(k) .
\end{aligned}
$$

But it is always possible to have $J^{\prime \prime}<J^{\prime}$ by appropriately choosing $\alpha>0$, and this always occurs since we are minimizing the performance index. In fact, since

$$
\boldsymbol{m}(k+1)=(1-\alpha) \cdot \boldsymbol{m}(k)=\boldsymbol{m}(k)+\Theta \cdot \boldsymbol{C} \cdot \boldsymbol{w}(k),
$$




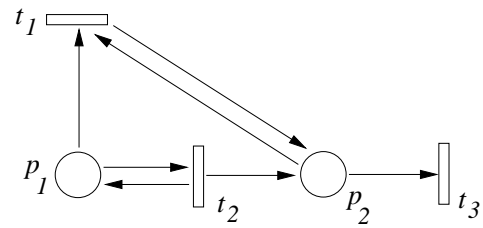

Fig. 4. A contPN that shows that the terminal equality constraint may not ensure stability when $N>1$.
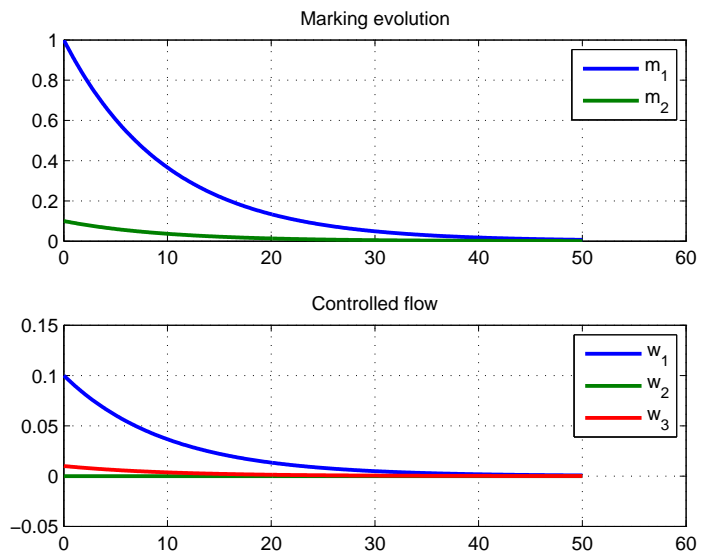

Fig. 5. Simulations for the net in Fig. 4 with $N=1$.

it follows that $\boldsymbol{m}(k)=-\frac{\Theta}{\alpha} \cdot \boldsymbol{C w}(k)$. Therefore

$$
\begin{aligned}
& J^{\prime \prime}-J^{\prime}= \boldsymbol{w}(k)^{T} \cdot \boldsymbol{R} \cdot \boldsymbol{w}(k) \\
&-\frac{\Theta^{2} \cdot(2-\alpha)}{\alpha} \cdot \boldsymbol{w}(k)^{T} \cdot \boldsymbol{C}^{T} \cdot \boldsymbol{Z} \cdot \boldsymbol{C} \cdot \boldsymbol{w}(k) \\
&= \boldsymbol{w}(k)^{T} \cdot \boldsymbol{R} \cdot \boldsymbol{w}(k) \\
&-\boldsymbol{w}(k)^{T} \cdot\left[\left(\frac{2}{\alpha}-1\right) \cdot \Theta^{2} \cdot \boldsymbol{C}^{T} \cdot \boldsymbol{Z} \cdot \boldsymbol{C}\right] \cdot \boldsymbol{w}(k)<0
\end{aligned}
$$

if $\alpha$ is small enough and $\boldsymbol{C}^{T} \cdot \boldsymbol{Z} \cdot \boldsymbol{C}$ is positive definite. But $\boldsymbol{C}^{T} \cdot \boldsymbol{Z} \cdot \boldsymbol{C}$ is always positive definite because $\boldsymbol{Z}$ is positive definite by definition and $C$ is a full rank matrix by assumption.

Remark 5.4: In general $\boldsymbol{m}(0)>\mathbf{0}$ is not a strict requirement in the above proposition. It is only sufficient to assume that for any $k \geq 0$ the optimization problem (plus terminal constraint) admits $\alpha>0$ as a solution. Physically this means that we can move along the straight line $\boldsymbol{m}(0)-$ $\boldsymbol{m}_{\boldsymbol{f}}$. However, since in general it is difficult to verify such a condition, for simplicity of presentation we prefer to state Proposition 5.3 providing a condition on $\boldsymbol{m}(0)$.

Note that in general the terminal equality constraint approach cannot be extended to the case of $N>1$ as long as the steady state values define an equilibrium point for the system when the final state + input is not a point interior to the set of feasible states + inputs.

Example 5.5: To illustrate this, let us consider the contPN in Fig. 4. Assume $\boldsymbol{m}_{\mathbf{0}}=\left[\begin{array}{ll}1 & 0.1\end{array}\right]^{T}>\mathbf{0}, \boldsymbol{m}_{\boldsymbol{f}}=\left[\begin{array}{ll}0 & 0\end{array}\right]^{T}$,
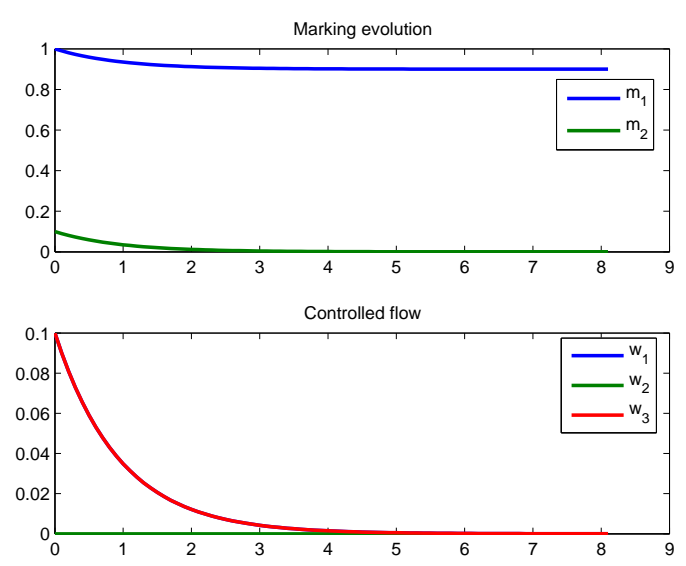

Fig. 6. Simulations for the net in Fig. 4 with $N=2$.

$\boldsymbol{w}_{\boldsymbol{f}}=\left[\begin{array}{lll}0 & 0 & 0\end{array}\right]^{T}, \boldsymbol{\lambda}=\left[\begin{array}{lll}1 & 1 & 1\end{array}\right]^{T}, \boldsymbol{Z}=\boldsymbol{R}=\boldsymbol{I}, \boldsymbol{Q}=\left[\begin{array}{llll}1 & 0 ; & 0 & 100\end{array}\right]$ and $\Theta=0.1$.

Applying MPC, and using the terminal constraint $\boldsymbol{m}(k+$ $N)=\alpha \cdot \boldsymbol{m}_{\boldsymbol{f}}+(1-\alpha) \cdot \boldsymbol{m}(k)$, we obtain the results in Fig. 5 and Fig. 6.

Thus, in the case of $N=1, \boldsymbol{m}_{\boldsymbol{f}}$ is reached (Fig. 5). On the contrary, $\boldsymbol{m}_{\boldsymbol{f}}$ is not reached if $N=2$ (Fig. 6). Observe that $\boldsymbol{m}_{\boldsymbol{f}}=\left[\begin{array}{ll}0 & 0\end{array}\right]^{T}$ is on the boundary of the feasible states since in a PN, $\boldsymbol{m}(k) \geq 0$ for all reachability markings $\boldsymbol{m}(k)$. The same happens for $\boldsymbol{w}_{\boldsymbol{f}}=\left[\begin{array}{lll}0 & 0 & 0\end{array}\right]^{T}$ that is on the boundary of the feasible inputs.

The results of Fig. 6 can be interpreted considering that if $N=2$ at each time instant the controller will choose to put "nothing" in $p_{2}$ at the first step and put "something" in the second step. Observe that to remove tokens from $p_{1}, p_{2}$ cannot be empty. But applying only $\boldsymbol{w}(0), t_{2}$ is never firing. This control is obtained because the performance index weights $p_{2}$ with a big value in the first step $(Q(2,2)=100)$ and with a small value in the second step $(Z(1,1)=Z(2,2)=1)$.

Note that if we consider $N=2$ but, instead of applying the resulting control law in the first time instant only, we also apply it in the second time instant, the closed-loop behavior of the net would be that reported in Fig. 7, i.e., the desired final marking is reached. Since in the first time instant the control is do nothing, i.e., $\boldsymbol{w}=0$, but in the next time instant it puts tokens in $p_{2}$, the control action varies from a non-zero to a zero value at every time instant.

Regarding the performance, it is obvious that this last case provides better performances than those obtained for $\mathrm{N}=1$. Indeed, a closed loop cost of 101.0058 is obtained in the case of $N=1$ while the cost is equal to 34.4362 if $N=2$ and both $\boldsymbol{w}(0)$ and $\boldsymbol{w}(1)$ are applied.

The above MPC control scheme is very conservative but ensures asymptotic stability even if the final marking and input is on the boundary. When this is not the case, i.e., final 

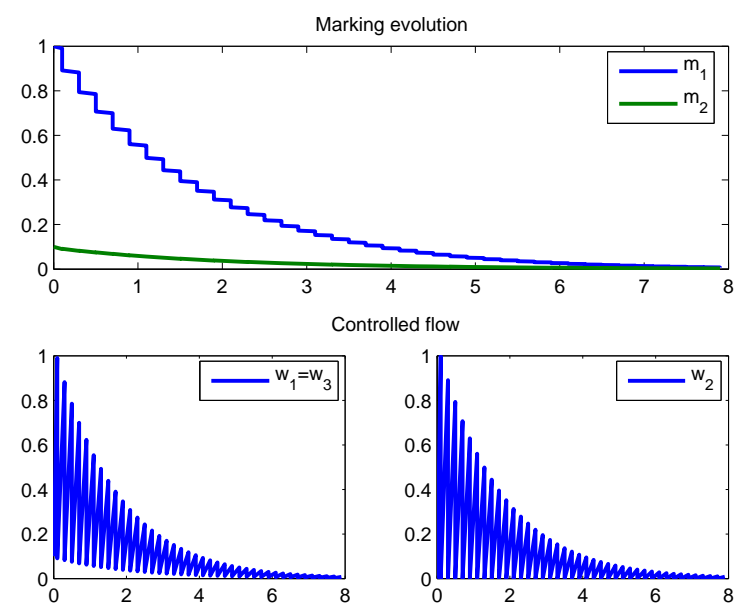

Fig. 7. Simulations for the net in Fig. 4 with $N=2$ and applying $\boldsymbol{w}(0)$ and $\boldsymbol{w}(1)$.

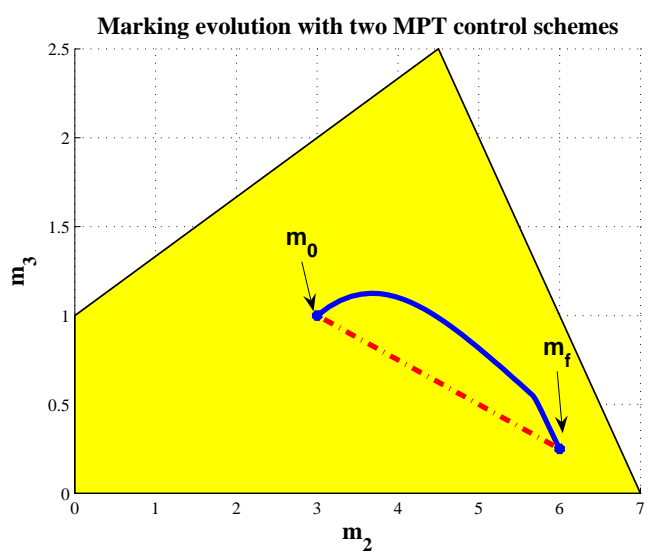

Fig. 8. Simulations for the net in Fig. 1.

marking and input is an interior point, the second method of ensuring stability (i.e., using unconstrained LQR for $j>$ $N)$ may be preferable because, in general, the closed loop performance index is better.

Example 5.6: Let us consider the net system in Fig. 2 with $\boldsymbol{m}_{0}=\left[\begin{array}{llll}3 & 3 & 1 & 3\end{array}\right]^{T}$. Let us assume that the desired marking is $\boldsymbol{m}_{f}=\left[\begin{array}{llll}0.75 & 6 & 0.25 & 8.25\end{array}\right]^{T}$ and $\boldsymbol{w}_{f}=$ $\left[\begin{array}{lll}0.125 & 0.125 & 0.125\end{array}\right]^{T}$. The reachability space projected on $\boldsymbol{m}_{2}$ and $\boldsymbol{m}_{3}$ is illustrated in Fig. 8. It can be seen that $\boldsymbol{m}_{f}$ is an interior point of the reachability space and it is easy to check that $\boldsymbol{w}_{f}$ is also an interior point of the feasible set of inputs. Let us assume $Z=100 \cdot \boldsymbol{I}$ for the MPC scheme with terminal constraints of the form (7), and $\boldsymbol{Z}=\boldsymbol{P}$, with $\boldsymbol{P}$ solution of (6), for the MPC scheme with unconstrained LQR; finally, let $\boldsymbol{Q}=\boldsymbol{R}=\boldsymbol{I}$. The resulting marking evolution is quite different in the two cases as it can be seen in Fig. 8. With continuous line it is represented the marking evolution with unconstrained LQR (approach
B2), and with dot line the evolution with terminal constraints (7). Under the considered assumptions, both schemes ensure asymptotic stability but the unconstrained LQR one gives a closed loop cost equal to 636.6636 instead of 717.641 obtained with terminal constraints (7).

\section{CONCLUSIONS}

We considered timed contPN under infinite server semantics. In previous works, on the basis of a constrained discretetime positive linear model of the system, we derived optimal control laws based on MPC. In this paper we analyzed some properties of the closed-loop system, such as feasibility and asymptotic stability. We proved that feasibility is always guaranteed, while asymptotic stability can only be ensured under certain restrictive assumptions.

\section{REFERENCES}

[1] A. Bemporad, M. Morari, V. Dua, and E.N. Pistikopoulos. The explicit linear quadratic regulator for constrained systems. Automatica, 38(1):3-20, 2002.

[2] A. Bemporad, M. Morari, V. Dua, and E.N. Pistikopoulos. Model predictive control of constrained piecewise affine discrete-time systems. Automatica, 38:3-20, 2002.

[3] A. Bemporad, F.D. Torrisi, and M. Morari. Performance analysis of piecewise linear systems and model predictive control systems. In Proc. of the 39th IEEE Conference on Decision and Control, pages 4957-4962, Sydney, Australia, 2000.

[4] S.L. Chung, S. Lafortune, and F. Lin. Supervisory control using variable lookahead policies. Discrete Event Dynamic Systems: Theory and Applications, 4(3):237-268, 1994.

[5] R. David and H. Alla. Discrete, Continuous and Hybrid Petri Nets. Springer-Verlag, 2004.

[6] A. Giua and F. DiCesare. Petri net incidence matrix analysis for supervisory control. IEEE Trans. on Robotics and Automation, 10(2):185-195, 1994.

[7] A. Giua, C. Mahulea, L. Recalde, C. Seatzu, and M. Silva. Optimal control of continuous Petri nets via model predictive control. In WODES'06: $8^{\text {th }}$ Int. Workshop on Discrete Event Systems, pages 235241, Ann Arbor, Michigan, USA, July 2006.

[8] J. M. Maciejowski. Predictive Control with Constraints. Prentice Hall, 2002.

[9] C. Mahulea, A. Giua, L. Recalde, C. Seatzu, and M. Silva. On sampling continuous timed PNs: reachability "equivalence" under infinite servers semantics. In $2^{\text {nd }}$ IFAC Conf. on Analysis and Design of Hybrid Systems, pages 37-43, Alghero, Italy, June 2006.

[10] C. Mahulea, A. Ramírez, L. Recalde, and M. Silva. Steady state control reference and token conservation laws in continuous Petri net systems. IEEE Trans. on Autom. Science and Engineering, 5(2):307320, April 2008.

[11] C. Mahulea, L. Recalde, and M. Silva. On performance monotonicity and basic servers semantics of continuous Petri nets. In WODES'06: $8^{\text {th }}$ Int. Workshop on Discrete Event Systems, pages 345-351, Michigan, USA, July 2006.

[12] B. De Schutter and T. van den Boom. Model predictive control for max-plus-linear discrete event systems. Automatica, 37(7):1049-1056, July 2001.

[13] P.O.M. Scokaert and J.B. Rawlings. Constrained linear quadratic regulation. IEEE Transaction on Automatic Control, 43(8):1163 1169, 1998.

[14] M. Silva and L. Recalde. On fluidification of Petri net models: from discrete to hybrid and continuous models. Annual Reviews in Control, 28(2):253-266, 2004. 